\author{
Virtual reality as a 'spiritual' experience: \\ A perspective from the cognitive science of religion \\ Northern Lights (2008): 6(1), pp 75-90 \\ Ryan G. Hornbeck \\ $\&$ \\ Justin L. Barrett \\ University of Oxford \\ Centre for Anthropology \& Mind \\ 64 Banbury Road \\ Oxford, OX2 6QS United Kingdom
}

Ryan G. Hornbeck is a D.Phil. student at the University of Oxford's Centre for Anthropology and Mind. He earned an undergraduate degree in social anthropology (B.A.) from Washington University in St. Louis and a M.Sc. in social anthropology from the University of Oxford. He has been researching social relationships in Second Life for over two years. Email: ryan.hornbeck@wolfson.ox.ac.uk.

Justin L. Barrett is Senior Researcher at the University of Oxford's Centre for Anthropology and Mind and is Lecturer in the Institute of Cognitive and Evolutionary Anthropology. He earned degrees in experimental psychology from Calvin College (B.A.) and Cornell University (Ph.D). Dr Barrett is a founding editor of the Journal of Cognition \& Culture and is a consulting editor for Psychology of Religion \& Spirituality. $\mathrm{He}$ is author of numerous articles and chapters concerning cognitive science of religion. His book Why Would Anyone Believe in God? (AltaMira, 2004) presents a scientific account for the prevalence of religious beliefs. E-mail: justin.barrett@anthro.ox.ac.uk.

Ryan G. Hornbeck and Justin L. Barrett 
Virtual reality $(V R)$ is often described as a gateway to a religious or spiritual experience - but why? In this article we hypothesize, using theories and evidence taken from the cognitive science of religion (CSOR), that human minds may interact with VR-hosted phenomena in a manner highly similar to that in which they interact with supernatural concepts.

Keywords: virtual, cognitive, religion, Second Life, touch

\section{Virtual reality as a 'spiritual' experience: a perspective from the cognitive science of religion}

Since the virtual reality (VR) boom of the 1990s, VR platforms and interactive worlds have been strong attractors for religious concepts. On the one hand, we see established religions, such as Christianity, Islam and Judaism using VR worlds as a place to congregate and evangelize (Grossman 2007). On the other hand, we often find that VR platforms are perceived as a gateway to a different, often 'spiritual', reality. Cyberpunk genre novels, many of them inspired by William Gibson's revolutionary and now classic Neuromancer (1984), frequently equate VR's immaterial properties to concepts of heaven and body transcendence (Wertheim 1999). Researchers working with sensoryimmersive VR platforms (the types with the headgear and motion sensors) have described VR as 'interactive mythology' (Rogers 1997) and 'mythological space' (Pesce 1997). Julian Dibbell, author of several popular books on VR and consultant to Linden Labs, creator of Second Life, was quoted in USA Today saying, 'virtual reality is in some ways an essentially spiritual experience' (Grossman 2007). Buddhist practitioners writing for websites such as http://www.secondseeker.com review the best VR sites for meditation. By these accounts and others considered below, it would seem that VR and religious concepts go quite well together - but why is this so?

In this article, we present theories and evidence drawn from cognitive science of religion (CSOR) that address this question. These findings might be of use to other researchers interested in the 'mediatization' of religion and the 'enchantment' of popular culture. Research in CSOR has shown that supernatural concepts the world over share 
certain properties with respect to the way they interact with the mind's social cognitive mechanisms and its natural and early-developed base of intuitive knowledge. In brief, supernatural concepts in religions contain information that (1) contradicts the intuitive set of expectations people bring to an ontological category of phenomena (i.e. natural objects, animals, etc.) and (2) allows people to draw a superabundance of inferences from their social cognitive mechanisms with minimal effort. In addition to briefly outlining some of these findings, we suggest that human minds may interact with VR-hosted phenomena in a manner highly similar to that in which they interact with supernatural concepts. Quite simply, VR representations frequently manipulate ontological norms, and VR world residents, enjoying anonymity and plasticity of representation, readily advertise the type of information that excites social cognitive mechanisms but which may be highly inappropriate or disadvantaging in the 'real'1 world. If religious concepts and VR-hosted phenomena do share properties that make them highly similar cognitive inputs, this might help to explain why VR is often referred to as a spiritual experience and, to a lesser extent, why it is a popular new frontier for religious evangelism. We then summarize these points by illustrating a common VR phenomenon - 'virtual touch' wherein counterintuitive representations and strategic information coalesce to create an emotionally salient experience that is itself counterintuitive and, by some accounts, spiritual-like. We intend these latter observations to serve (rather tentatively, we realize) as a preliminary empirical footnote to what is otherwise a conceptual analysis. Finally, we conclude by suggesting that if VR worlds can generate experiences that are subjectively referred to as 'spiritual', then such worlds can be a productive laboratory for CSOR researchers and media theorists alike.

Throughout this article we frequently use Second Life (SL) as a base of reference for our observations about VR. Though VR technically refers to any technology that allows a user to interact with a computer-simulated environment - this could be anything from a chat room to a sensory-immersive CAVE platform - we have selected SL for several reasons. For one, SL's popularity makes it representative of many people's

\footnotetext{
${ }^{1}$ Social anthropologist Tom Boellstorff has pointed out that using the term 'real' to describe non-computer mediated interactions is problematic in that it falsely presumes there is nothing 'virtual' about such interactions (Lattin 2007). We consider this to be a valid point but we have retained the use of 'real', by which we mean non-computer mediated interactions, in order to minimize confusing language and theoretical asides.
} 
perceptions of VR. SL currently hosts over eight million open accounts and approximately 40,000 residents are online at any given moment. For another, SL's impressive graphic and interactive capacities make it one of the most potentially immersive (i.e. 'otherworldly') VR worlds created to date. Finally, because SL is entirely imagined, created and owned by its users, the content therein reflects a relatively democratic expression of human cognition, as opposed to heavily themed content designed by a team of professional developers. With these reasons in mind and in order to prime the reader with a better understanding of VR and VR-based relationships, we wish to preface the main body of this article with a brief introduction to SL.

\section{Introduction to Second Life}

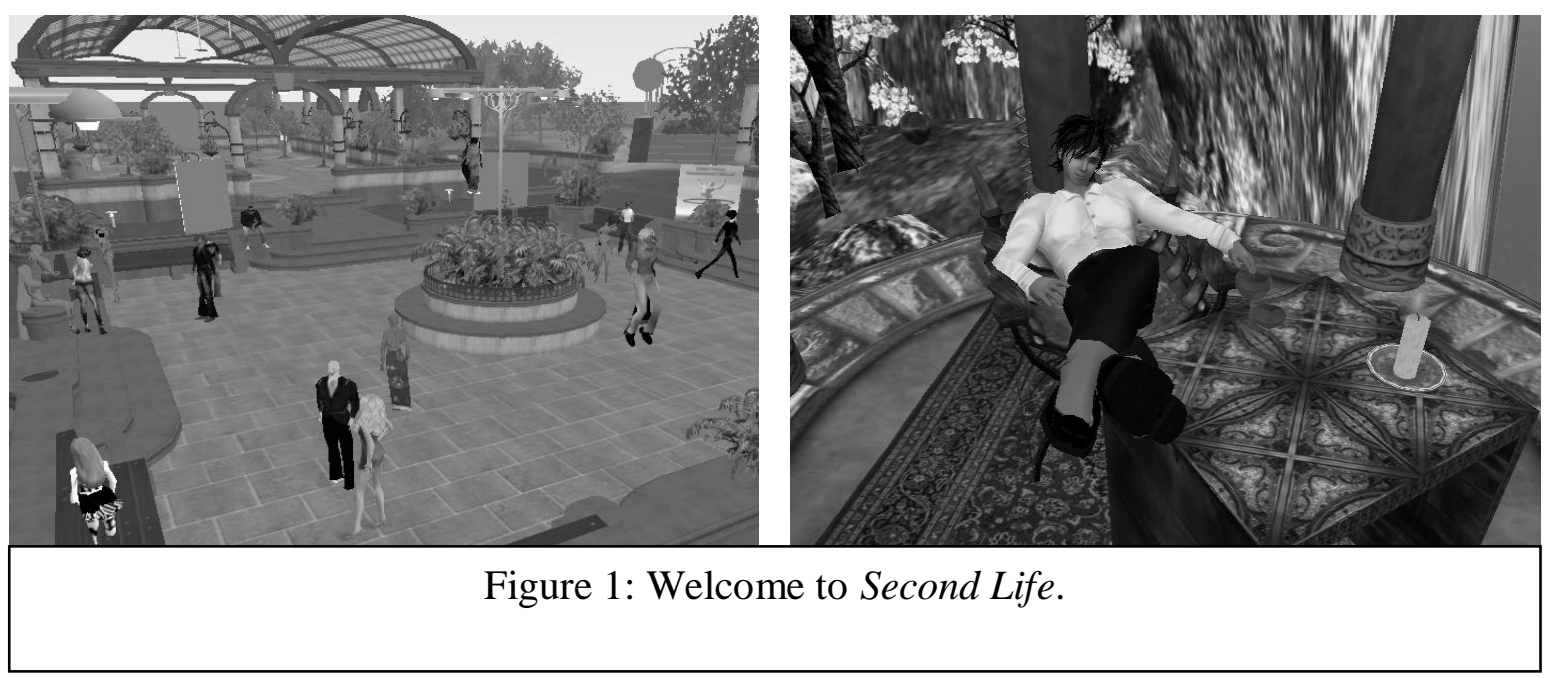

Every day, people all over the world interact with each other in SL, a VR world that is entirely imagined, created and owned by its users. Residents inhabit SL in their virtual bodies - their 'avatars' - humanoid or otherwise, which they construct with limits set only by their imaginations and artistic abilities using SL's built-in content creation tools. Unlike massively multiplayer online role-playing games (MMORPGs), such as the popular World of Warcraft, SL has no objectives or pre-programmed activities. Rather, SL simply encourages residents to recreate their lives 'in-world' - to find a means of deriving an income (SL boasts a fully integrated economy that transacts over one million US dollars - real money - per day), buy a cosy virtual home, fall in love and socialize with fellow residents. Indeed, there are numerous venues in which to socialize. SL is 
filled with museums, beach parties, shopping malls, research institutions, picturesque gardens, support groups and worship services. Management consultants meet and give presentations on private islands. Directors film movies in SL and exhibit them at SL film festivals. Charity organizations host galas and raise thousands of US dollars for various causes. And so on.

Avatar interactions in SL feature prominently in this analysis, so a little background might be helpful. SL residents are situated in SL by way of an avatar, which serves as a resident's 'zero point' of perception and social contact. One can go a little outside, but cannot move entirely beyond one's avatar. For example, one cannot be viewing books in a SL library while one's avatar is soaking up rays on the beach. Resident and avatar are inextricable, and so the latter is an important part of one's online identity. Consequently, great amounts of time, effort and money go into avatar construction. Avatars are almost infinitely malleable; and for those who lack the artistic talent or software skills to develop their own appealing avatar, hundreds of privately owned boutique shops trading in body shapes, skin colours, hairpieces and even genitalia enable residents to look their best.

These avatars communicate with one another in various ways. The most basic form of communication is text based. Typed messages may be sent privately or displayed to all avatars in a general area. Avatars may also create or buy gestures; these animate the avatar into a themed sequence of movements, such as 'wave hello' or 'roaring laugh'. Or, avatars may be animated into certain motion sequences by clicking on pose balls, which initiate a sequence of animations (frequently used for dancing and cuddling) that is circuitous and continues until the user(s) wishes to stop. Lastly, SL has recently gained voice-over capabilities. These enable a resident to speak in real time with other residents.

\section{The cognitive appeal of supernatural concepts}

CSOR research shows that supernatural concepts tend to cluster around phenomena that (1) contradict the intuitive set of expectations we bring to an ontological category of those phenomena (i.e. natural object, animal, etc.) and (2) allow us to draw a superabundance of inferences from our social cognitive mechanisms with minimal effort. Starting with point (1), we might begin to explicate these findings by way of a simple 
question: do you find it requires more mental effort to solve ' $345 \times 729=$ ?' or to recognize your cousin at an annual family reunion? Most of us would agree that multiplying three-digit figures is more difficult than recognizing a family member, even if we have not seen him or her in a few years; but why is this so? What is happening in our minds that we can process the minutiae of one's facial details and match those details to an image set down in memory a year ago, yet a multiplication problem taken from an elementary school textbook requires paper, pencil and time? Why are our minds more receptive to some forms of information than others?

Cognitive scientists studying the way human minds organize and develop during childhood generally agree that either from birth or during childhood human minds develop numerous functional subsystems that carry out particular information-processing tasks (Pinker 1997). These functional units operate with such fluency and automaticity, especially when performing tasks that are fundamental to human survival, that we often process them without conscious effort or awareness. To illustrate, for most humans recognizing familiar faces requires no studying of features or deliberate attention to detail; rather, this task is automatic.

Along with this automaticity, or what might be called cognitive naturalness (McCauley, forthcoming), come certain cognitive biases. That is, within a given domain our minds automatically assume certain kinds of relationships and tend towards certain kinds of understandings. Again, this intuitive knowledge makes good sense in terms of survival. For instance, developmental psychologists have demonstrated that within the first few months of life babies provide behavioural evidence (typically looking behaviours) indicating that they understand that physical objects (1) do not pass through one another, (2) must move as cohesive wholes, and (3) must move continuously from one place to another (instead of disappearing and reappearing) (Spelke and Kinzler 2007). These expectations of physical objects can be understood as intuitive knowledge in the sense that people intuitively assume it is true of any object encountered. Experimental evidence suggests that humans routinely acquire domain-specific intuitive knowledge concerning - and sometimes specific to - a range of ontological categories for things as ordinary as physical objects, artefacts, animals and other living things, and intentional beings such as people (Sperber, Premack and Premack 1995). 
Cognitive scientists studying cultural expression have found it productive to ask how the intuitive knowledge generated by cognitive systems informs and constrains the generation and transmission of cultural expression. For instance, the psychologist, Thomas Ward, discovered that when trying to create novel extraterrestrial beings, adults' creations were highly constrained by the assumptions of our intuitive ontological knowledge concerned with living things. Novel beings overwhelmingly exhibited bilateral symmetry and at least one pair of major sense organs and limbs, even when adults were explicitly told to be as creative as possible and not worry about believability (Ward 1994, 1995). Similarly, the psychologists Michael Kelly and Frank Keil (1985), found that the metamorphoses in the writings of Ovid and Grimm's Fairy Tales were not arbitrary or uniformly distributed over theoretically possible types of transformations, but appeared to be closely constrained by intuitive cognitive systems. Transformations to 'neighbouring' ontological categories (based on childhood concept formation) appeared to be more effective story devices than other types of transformations.

Perhaps the most developed area in this cognition and culture field is CSOR. In the area of religious expression, too, scholars have suggested that culturally successful ideas must closely conform to the intuitive expectations or biases of early developing cognitive systems. Such intuitive ideas are more likely to be spontaneously produced and successfully communicated because their elements already lurk in human conceptual systems. But being completely intuitive is not always a good thing. When competing for human attention, the intuitive can be overlooked. Intuitive is often boring. Cognitive scientists of culture have noted that better than being wholly intuitive is being slightly counterintuitive (Barrett and Nyhof 2001; Boyer 2001; Sperber 1996).

Compare the idea of a cat that has kittens with the idea of a dog that has kittens. As much as one might enjoy a moving tale about a cat having kittens, hearing about a dog having kittens is likely to be much more striking and worthy of retelling. Why? The anthropologist and psychologist Pascal Boyer suggests that it is because one version is wholly intuitive and the other modestly counterintuitive. Research has shown that young children intuitively know that animals have offspring of their own kind, passing on those features that have biologically functional consequences (Keil 1989; Springer and Keil 1989). A dog having kittens is thus counterintuitive, in this technical sense, and more 
striking than other unusual arrangements. Experiments demonstrate that concepts with a single counterintuitive feature are better remembered and communicated than comparable wholly intuitive concepts (Barrett and Nyhof 2001; Boyer and Ramble 2001).

These technical senses of intuitive and counterintuitive allow for a concept to be intuitive in two ways. First, a concept may be specified by naturally developing cognitive systems; for example, that solid objects cannot pass through other solid objects appears to be a default assumption of natural cognitive systems. The idea of a brick not being able to pass through a tree is intuitive because it conforms to default assumptions. A second way in which an idea may be intuitive is by simply not violating expectations. The idea of a 100-pound rodent is intuitive (even if surprising) because natural systems do not specify how much rodents can or cannot weigh.

A clarification may be helpful at this point. In keeping with Boyer's and others' previous research in this area, we reserve the term 'counterintuitive' for ideas that violate naturally developing intuitive knowledge, what McCauley (forthcoming) calls 'maturationally natural' cognition. That is, cognition that develops as part of normal human maturation regardless of cultural settings. Nevertheless, with special cultural aids such as explicit instruction, opportunity for rehearsal and supporting artefacts, individuals may acquire tremendous cognitive fluency in particular areas. McCauley dubs this culturally dependent kind of cognitive mastery 'practiced naturalness'. Consider, for instance, chess or calculus mastery. Scripts for social interactions (such as what to do when ordering at a restaurant) or schemata (such as the expected temperament of different breeds of dogs or the size limits of rodents) exemplify the sort of individually or culturally specific conceptual structures that arise through practised naturalness. We might term ideas that violate these practiced natural expectations counterschematic. Counterschematic ideas, then, violate expectations that have arisen through practised natural systems and are analogous to counterintuitive ideas that violate expectations that have arisen through maturationally natural systems. To illustrate this, asking for the bill at a sit-down restaurant before the ordered meal has arrived could be counterschematic but not counterintuitive. The idea of a 100-pound rodent or a high-strung Labrador might be counterschematic, but neither is counterintuitive. Whether or not something is 
counterschematic is individually and culturally variable. Whether or not something is counterintuitive is a matter of normal human development.

Concepts that are counterintuitive can be more attention demanding, but this advantage is not without limits. Rather, Boyer (2001) suggests an optimum. Concepts that are mundanely intuitive are acceptable candidates for being successfully spread within a population; those with just a minor counterintuitive tweak are better; and those with too many counterintuitive features are disastrous. Consider a liquid dog that gives birth to invisible artichokes made of cardboard whenever someone thinks about something that will happen next Tuesday. Such a dog concept (if indeed it can be called a concept) so greatly violates the intuitive conceptual structure that it is hard to understand and difficult to communicate effectively without distortion. Furthermore, its counterintuitive structure renders it cumbersome for generating predictions, explanations or inferences. A large, brown dog with floppy ears, a white-tipped tail and a pleasant disposition that gives birth to kittens does not present the same conceptual challenges for its communication or for its ability to generate predictions, explanations or inferences.

Applying these cognitive insights to supernatural concepts, Boyer and his colleagues have argued that the vast majority of supernatural concepts that feature in religious belief systems across cultures have a number of defining features (see, for example, Atran 2002; Barrett 2004; Boyer 2001, 2003). First of all, they tend to be minimally or modestly counterintuitive; that is, one or two intuitive expectations are violated. For example, a statue that can listen to petitions is an artefact to which has been added the property of having a mind. Forest spirits in some places might be characterized as essentially invisible people - invisibility being the single important counterintuitive feature. A second common feature of religious concepts is that their counterintuitive feature enhances their inferential potential in important realms, such as survival or social interaction. By inferential potential we refer to these concepts' abilities to rapidly generate further ideas, inferences, explanations and predictions, particularly about those things that matter to us.

We can be more clear here about 'inferential potential' and 'those things that matter to us' by way of a brief introduction to evolutionary psychology and, specifically, the importance of our unique, evolved social cognitive mechanisms. These social 
cognitive mechanisms ${ }^{2}$ enable our species to communicate and cooperate in a manner that is unique in scope and complexity. Relative to other species common to our evolutionary milieu - the African savannah of Pleistocene onwards - humans are in many respects an outmatched class. Humans are not particularly fast or strong, they cannot easily navigate treetops, and they have no protective covering. Their large brains create birthing hazards, require additional time to develop and consume a disproportionate share of nutritional resources. Without the unique set of cognitive abilities that enabled the formation of cooperative bonds and the transmission of accumulated information, humans would never have enjoyed such spectacular evolutionary success.

To illustrate this, one such social cognitive mechanism, 'theory of mind' (ToM), refers to the human mind's unique ${ }^{3}$ ability to form assumptions about another person or animal's mental state. 'Theory', in this case, refers to our ability to postulate, or form a theory about, what other people might be thinking. For example, if a friend stops waving to me as I pass him on my daily commute to work, I might assume that in his mind he is angry with me. Or, if a co-worker's promotion was mentioned in an e-mail to all employees, when I go to congratulate her I will assume that she knows that I know she was promoted. Here I am not just theorizing about her mental state, I am also theorizing about what her mind might be theorizing about my mind. This is an instance of what we would call 'second-order' ToM. An instance where Jack assumed that Jane thought that Bob suspected Linda of thievery would be an instance of third-order ToM.

These multiple-order ToM exercises represent the day-to-day conditions of social interaction, that is, the conditions contributing to prosperity or failure. We are constantly stressing about and trying to gain access to the information that others have about us.

\footnotetext{
2 By 'system', 'mechanism', or 'device' we refer to a functional unit of the brain that may or may not be driven by a particular localized section of the brain devoted to a particular task. Our mental hardware for vision, for example, is mostly located in six specific areas (V1 to V6) of the occipital lobe. Even where the location, or locations (tasks may be highly distributed), of the system may be debatable, we may be relatively certain that some task-centric bounded entity exists in the mental hardware because, as the following examples show, some persons who have sustained head injuries or abnormal brain development have lost the ability to perform the particular task in question (though all other brain functions may be unaffected).

${ }^{3}$ Neither autistic humans nor primates seem to share our ToM capacities, though the status of a chimpanzee's theory of mind is contentious. Some researchers deny chimps have provided any clear evidence of ToM capacity (Povinelli and Vonk 2003), whereas others believe chimps have rudimentary ToM (Tomasello, Call, and Hare 2003). Nearly all scholars in this area agree that chimps are not capable of the ToM capacities of three-year-old children and certainly do not reach third-order ToM.
} 
'Does my boss think I am doing a good job?' 'Was Jason suggesting that Michael is out to get me?' These are important questions. And tens of thousands of years ago, figuring out who was of a cooperative mind and who was liable to cheat was a daily matter of life and death. Given the importance of ToM in both our evolutionary history and contemporary circumstances, these types of thoughts enjoy privileged access to the conscious realm of our minds. Even seemingly non-related tasks often feed back into ToM, such as when a child practises her algebra so that her parents might form positive mental representations of her. Consider also that ToM exercises that are decoupled from the consequences of real social interaction make for fascinating, emotionally charged entertainment. People all over the world love to exchange gossip about what some people think about other people (Gambetta 1994; Haviland 1977). Fans of detective thrillers and Shakespearean dramas delight in figuring out who has access to what information and what inferences can be drawn from that information (Dunbar 2004; Stiller, Nettle and Dunbar 2004). In fact, ‘dramatic irony’ by definition occurs when the audience has important information that the characters lack. That we pursue these exercises even when there is nothing practical to be gained points to another important characteristic of social cognitive mechanisms - they are often accompanied by strong emotional incentives. ${ }^{4}$

Given, then, its importance in our evolutionary history and its access to powerful emotional states of mind, ToM is always tugging at the conscious realm of our mind, looking for inputs - or strategic information - that will help ToM produce helpful inferences about what is going on in other people's minds. We might briefly examine the relationship between these two: first, what qualifies as strategic information will differ for each person and circumstance. For example, the information that you have a new, expensive, red mountain bike in your garage is not necessarily strategic to me, unless I am missing such a bike. If I am missing such a bike, the information becomes strategic and is seized upon by my social cognitive mechanisms, which generate inferences that motivate and guide my next actions. With respect to ToM, I now infer that you might be harbouring other uncooperative thoughts in your mind. I am now angry or fearful and

\footnotetext{
${ }^{4}$ By emotional incentive, we refer to those biochemical processes that result in mental states that are commonly referred to as love, sadness, anger, jealousy and so forth. From an evolutionary perspective, these biochemical processes and their associated mental states were crucial in motivating and guiding our behaviour to sustain important social relationships (Fessler and Haley 2003; Fiske 2002), especially when these required action that was inconsistent with our immediate self-interest.
} 
well motivated to take precautions to safeguard against your anti-social tendencies. In summary, our minds are always receptive to information that enables us to infer what others are thinking about us, because getting this information was crucial to our survival in our evolutionary past.

This brings us back to the second common feature of religious concepts - their counterintuitive feature enhances their inferential potential in realms important to humans, such as survival or social interaction. Gods always seem to know more than people do - particularly about the intentions of others or which herbs in the forest heal us, rather than human intestinal length or how many frogs live in Colombia. Sometimes gods have access to this strategic information by virtue of super knowledge or super perception, but others gain it through invisibility or counterintuitive spatial or physical properties (Boyer 2001). Supernatural entities could be counterintuitive by virtue of vanishing every new moon or experiencing time backwards, but these counterintuitive properties do not stimulate interesting questions and speculations about who might know what I did last night with whom. ${ }^{5}$

In summary then, supernatural ideas are distinguished by the special use they make of our ordinary cognitive capabilities. They get our attention and stimulate our naturally developing cognitive systems by (1) striking a balance between understandable intuitiveness and attention-grabbing novelty; and (2) including properties that enhance (rather than detract from) inferential potential in matters of importance, particularly social ones. These two properties give supernatural concepts in religious systems cognitive appeal - they demand attention, support conceptual exploration and often generate strong emotional experiences. We suggest that these two features also factor prominently in VR worlds and suggest a reason why participants in SL and other virtual spaces often compare their experiences to the spiritual and religious. From a cognitive science perspective, SL is populated by analogues of supernatural beings and prompts pseudosupernatural experiences. But how exactly is this so?

\footnotetext{
${ }^{5}$ Access to strategic information is also part of what separates gods and spirits from counterintuitive but decidedly non-religious concepts, such as a cartoon animal that can speak English. Aside from not being postulated to exist or act in the real world, counterintuitive fictional characters have little inferential potential in day-to-day activities.
} 


\section{The cognitive appeal of VR inputs}

Consider first that SL is replete with counterintuitive representations. An avatar, for example, preserves most of the same expectations we would have of a real person: it has a specifiable location in space and time, self-awareness and expressive mental states, moves in goal-oriented ways and understands language and communication. Also, avatars interact in a manner that is largely intuitive (even if a bit surprising): they gossip, play sports, attend social events, engage in intimate behaviours and so forth. Yet an avatar, even one that looks strikingly real, also presents a few critical violations. The most obvious of these is that avatars lack materiality. Avatars can go through walls (if the VR physics generator permits), move objects without coming into contact with them, disappear, fly and radically alter their anatomy.

This immaterial property of VR simulations has long fascinated VR enthusiasts, who frequently equate this property with religious and spiritual concepts. In Gibson's Neuromancer (1984), the protagonist, Case, is at one point banished from the digital paradise of cyberspace (an event referred to as 'the Fall') and condemned to a life of 'imprisonment' in the flesh (i.e. embodiment and its corporeal restrictions). Fortunately for Case, in the end he regains 'the bodiless exaltation of cyberspace' (Gibson 1984: 6), and presumably a release from those intuitions predisposed to a material world, when his digital form is fed into the matrix to live forever in cyber-paradise. Gibson's next protagonist, Bobby Newark, achieves a similar end in Mona Lisa Overdrive. In fact, this vision of body transcendence is a popular theme throughout the cyberpunk genre. Journalist and non-fiction author, Margaret Wertheim, summarizes great tracts of this genre when she observes that 'nothing epitomizes the cybernautic desire to transcend the body's limitations more than the fantasy of abandoning the flesh completely by downloading oneself to cyber-immortality' (Wertheim 1999: para. 10). Wertheim suggests that this desire reflects the cyberpunk belief that 'at core a human being is reducible to an array of data' (Wertheim 1999: para. 15), and this separation of the person or self from the body shares much with the cross-culturally recurrent religious notion that a soul or spirit may be separated from the body (for example, Cohen (2007); see also Bloom (2004) for an account of dualism's origins in human cognitive development). 
This association with religious phenomena makes even more sense when we consider that immateriality enhances one's access to strategic information by enabling an analogue of the super perception enjoyed by so many ancestors and deities. Without material constraints, avatars can teleport instantly to any location in SL, see through walls and across great distances, and become invisible. Like many gods and spirits, they can be anywhere at any time and can evade detection.

Additionally, SL is teeming with strategic information that is not necessarily built into, but is nonetheless associated with, these counterintuitive representations. Computer mediated communication (CMC) researchers and theorists have long noted that online relationships, particularly romances, are often vulnerable to a 'boom or bust phenomenon' (Cooper and Sportolari 1997). This phenomenon occurs where a rapid process of self-disclosure leads budding relationships to develop at a highly accelerated pace towards success or failure. For CSOR researchers, this 'boom or bust phenomenon' might seem to be what naturally happens when social cognitive mechanisms are superstimulated. Given access to plentiful stores of strategic information, i.e. a potential mate's preferences, values and so forth, our social cognitive mechanisms are able to process dispositions towards others much more quickly than they could in the real world, where strategic information is hard to acquire. We can identify at least two mechanisms of this phenomenon in SL.

First, most residents in SL observe strict boundaries between SL and what they frequently refer to as ' 1 st' life. Simply clicking on the '1st Life' tab of a resident's profile often produces a pithy defence of anonymity such as 'Don't ask.' Many theorists have commented on the affordances gained by this anonymity. Lea, Spears and DeGroot (1995: 202), for example, have noted that:

The visual anonymity of the communicators and the lack of co-presence - indeed the physical isolation - of the communicators add to the interaction possibilities, and for some this is the 'magic' of on-line relationships.

Undoubtedly, some of these 'interaction possibilities' and part of this 'magic' also resides in the immunity to negative consequences that might be suffered for being too forthcoming with strategic information in the real world. For example, to blatantly 
advertise oneself as a potential sexual partner in the real world could be quite damaging to one's social status. But in SL, such information might be displayed on one's profile, as might one's values, accomplishments, scandals and so on. With anonymity, people can (and do) do whatever they please, and this includes trade freely with pieces of strategic information.

Second, SL's content creation tools enable a resident to shape visual, audible and, if using a haptic interactive device, tactile, rhythmic or proprioceptive information into strategic information. Evolutionary psychologists have noted that some physical traits tend to be highly sought after when selecting mates. Women are usually attracted to men approximately 6 inches taller than themselves and who show physical signs of dominance such as musculature and a square jaw (Ellis 1992). Among other features, men value youthful appearance - younger than themselves - (Buss 1989) and facial symmetry (Grammer and Thornhill 1994). Theoretically, these features suggest a high degree of evolutionary fitness to a potential mate, who of course wants to maximize his or her progeny's chances for survival. In SL, one may design one's avatar in accordance with these features or any other visual, audible or tactile properties that are likely to be seized upon by one's social cognitive mechanisms.
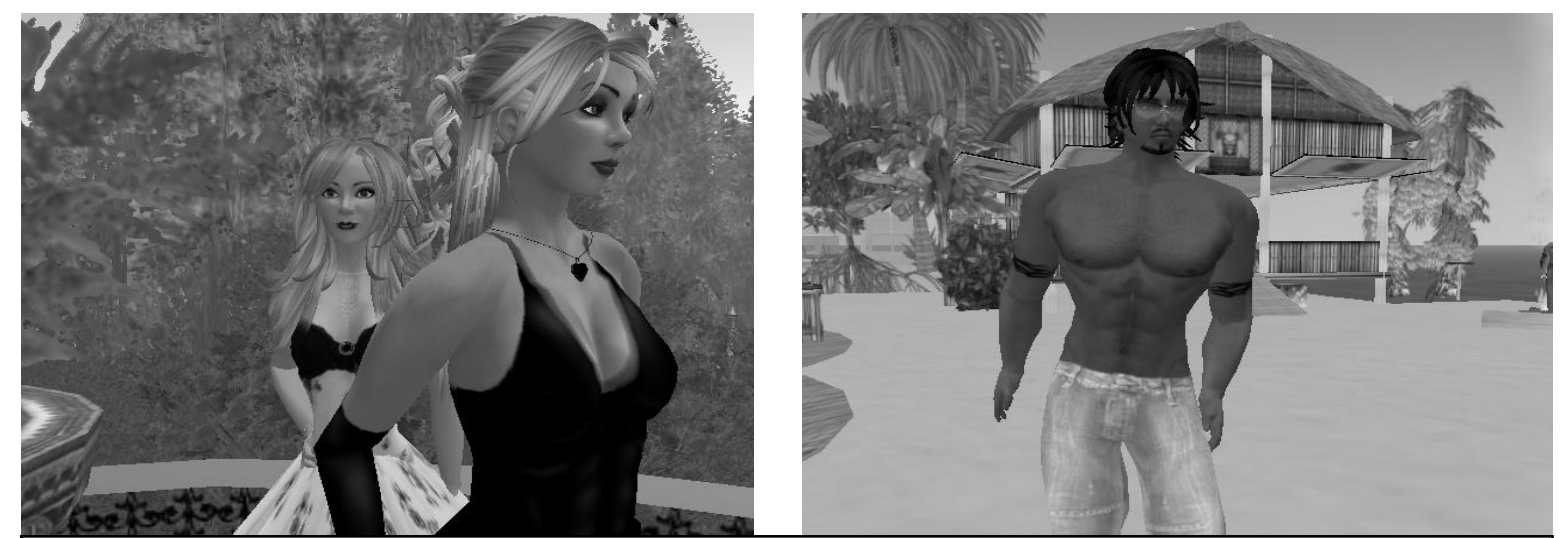

Figure 2: Strategically proportioned avatars.

VR concepts, then, like supernatural concepts, are distinguished for making special use of ordinary cognitive capabilities. They get our attention and stimulate our naturally developing cognitive systems by (1) striking a balance between understandable 
intuitiveness and attention-grabbing novelty; and (2) including properties that enhance (rather than detract from) inferential potential in matters of importance, particularly social ones. These two properties give VR concepts cognitive appeal - they demand attention, support conceptual exploration and can be accompanied by powerful emotional experiences. However, it should also be clear that VR concepts do not work at an optimum. They are weak analogues, not functional equivalents, of those supernatural concepts that feature in popular religions. It is possible that focusing on this gap in affective content could be productive for both CSOR researchers and media theorists. We will return to this suggestion in our conclusion.

\section{Virtual touch: a case of combining counterintuitive representations and social inferences}

As a tentative empirical footnote to these observations, we wish to illustrate a phenomenon in SL (we have termed it 'virtual touch') in which the two inputs discussed here (counterintuitive representations and strategic information) combine to produce an experience that might resonate as supernatural or 'spiritual-like'.

In previous examples, strategic information was visual (Y can see $\mathrm{X}$ 's treachery) or audible ( $\mathrm{Y}$ hears that $\mathrm{X}$ has a crush on her); but it can also be kinaesthetic. Consider the satisfaction one feels when one receives a hug or a caress from a loved one. The pleasure of touch is no accident; it is, rather, an important part of our evolutionary heritage. Anyone who has been to the zoo knows that chimpanzees spend a lot of time grooming one another. This is not just idle folly on the part of the chimpanzee. Rather, chimpanzees are social animals that depend on cooperative bonds for survival; and because chimpanzees have no language, touch is an extremely important form of communication. When one chimpanzee removes parasites and other detritus from the back of another chimpanzee, the groomed chimpanzee registers a spike in endorphins (Dunbar 2004). It feels good to be touched and groomed, and these good feelings provide a strong emotional platform for cooperative bonds. It is the same with humans. Gently touching a human infant's body releases oxytocin, a 'feel good' hormone that predisposes the infant to develop an attachment to the mother and is essential to the infant's proper mental and bodily development (Uvnas-Moburg 1998, 2003). This relationship between 
touch, pleasure and social cooperation persists in adulthood and has been demonstrated in many studies (Hertenstein, Holmes, Kerestes and Verkamp 2006). It follows that touching someone is an embodied and powerful form of social cognition because it can catalyse oxytocin and predispose the recipient to think in a certain, social way - that is, affectionately and cooperatively - about his or her peers. And touching, like many forms of strategic information, is much more regulated in the real world than it is in SL.

Now consider that SL avatars are constantly touching one another through the use of pose balls and animations. Lovers stroll through a park arm-in-arm. Churchgoers hold hands during a worship service. Friends greet one another with a hug, a slap on the back or perhaps a kick to the chest. A common but curious feature of these activities can be found in reports of extended embodiment by way of tactile sensations registered through the avatar. That is to say, if one's avatar is touched on the arm or chest, one sometimes registers tactile sensations on the corporeal arm or chest. Consider these examples, taken from various blogs and websites, in which SL residents report or suggest corporeal sensations and oxytocin-like benefits (all interactions described are between avatars in $\mathrm{SL)}$ :

The SL hug is a high fidelity representation of the actual experience, and even though no one would confuse the virtual hug with the real one, the virtual one will still make you respond in the same way - it evokes a remarkably warm and fuzzy 'feeling' of being hugged. (Johnson 2007)

The [sadomasochism] desk allows an animation where he can caress my ass before spanking it. Then seeing his hand rise and fall with the blows ... Ohhhhh *shivers and grins* ... it's very very sensual. (Horton 2007)

Odd how all of SL fades when we are together - that the 'touch' of another avatar is better than the sweetest singer or most breath-taking view; that a kiss or caress distracts me from my need to dance or travel. (Website is no longer available)

'It could get pretty hot and erotic at times,' Farrant said. 'You see your avatar placing your hands on another avatar, which is a very sensual thing.' (Machalinski, McConnon and Nicholson 2006) 
SL hugs warm the heart - OK not like a real life hug, but that person still touches you.

(Viklund 2007)

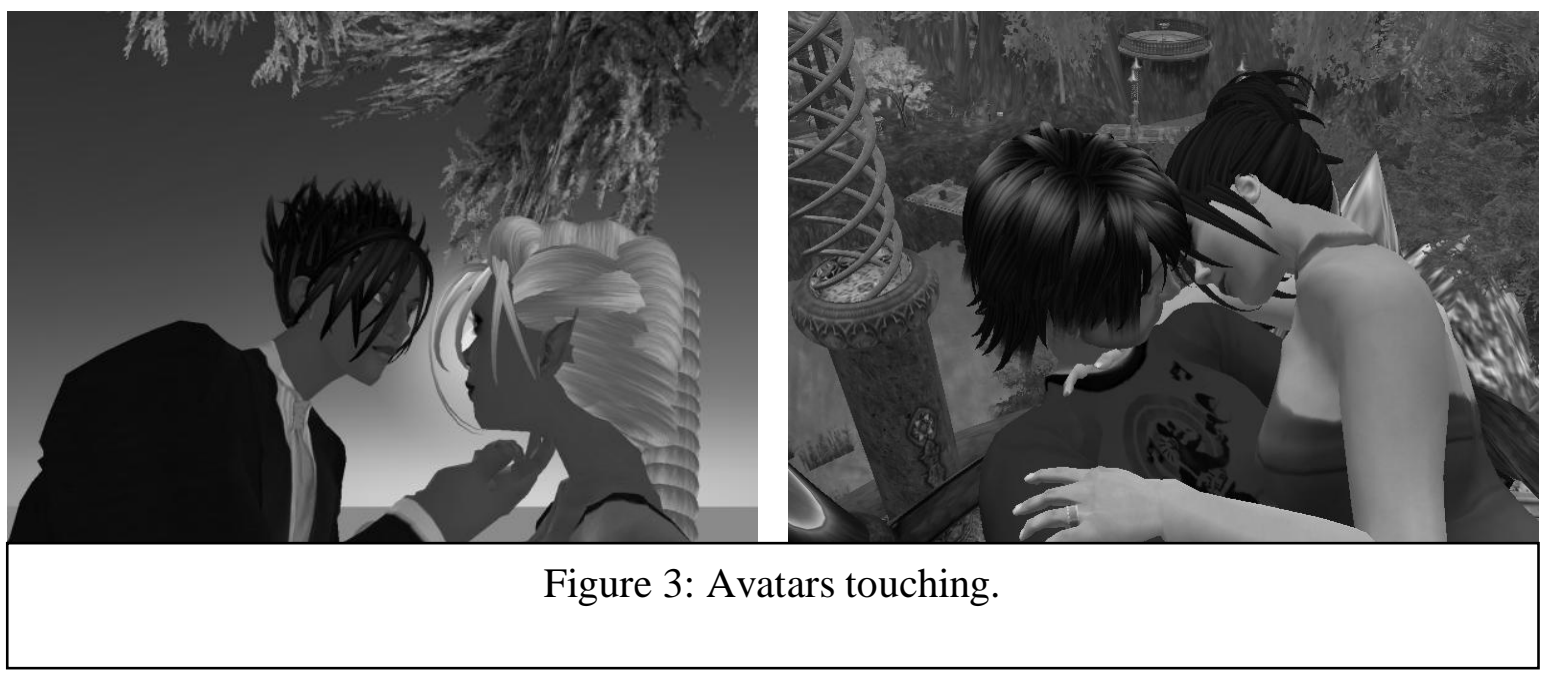

These reports have been analysed elsewhere for what they can tell us about the cognitive foundations of instances of extended embodiment (Hornbeck 2007). Here we simply wish to note that SL residents who were highly focused on VR phenomena (for example, for more information on 'presence' or 'being there' in VR worlds, see Garau, Slater, Pertaub and Razzaque (2005) and Yee (2005)), which included counterintuitive representations (avatars, VR terrain) and strategic information (touching and any profile, visual or other information), reported an emotionally salient experience - corporeal sensations and 'warm', oxytocin-like effects - that was itself counterintuitive! That is, to register tactile sensations where no material agent has administered them violates intuitive expectations of the relationship between touch and sensation, and might by some accounts be considered a 'spiritual-like' experience by virtue of this counterintuitiveness. If something remotely 'spiritual-like' can be imparted by technology that is very tame in comparison to those other worlds represented in cyberpunk literature, future VR developments could inspire experiences that are, by all accounts, religious or spiritual.

\section{Conclusion}

Making special use of ordinary cognition seems to characterize many religious concepts and experiences as well as many VR concepts and experiences. Specifically, religious 
cognition and VR cognition only modestly violate intuitive cognitive expectations in such a way as to yield unusually high levels of inferential potential, particularly in social domains. Perhaps because of these parallels, for some the conditions already seem sufficient to qualify VR experiences as quasi-religious or spiritual. This is potentially significant. For CSOR researchers, VR suggests a new way of examining the mind's resources. Cognitive scientists have long recognized that it can be productive to look at brain-damaged patients who have lost the capacity to perform certain tasks - to look at how the system responds when something has been knocked out (Gazzaniga, Velletri and Premack 1971). In VR worlds such as SL, on the other hand, where we can retain control over phenomenal inputs, we may proceed by examining what has been put into the user. Which conditions suffice for a religious or spiritual experience, and which do not? Perhaps by using VR and borrowing from media theorists specializing in religious content, we can approach these questions with greater precision. For media researchers, examining this gap in affective content and how this gap narrows or widens with new technologies could help to explain how VR, and media generally, contributes to the 'mediatization' of religion and to the 'enchantment' of popular culture. The CSOR material we have presented here is only a brief introduction to a large body of work that could be valuable to those researchers. 


\section{References}

Atran, S. (2002), In Gods We Trust: The Evolutionary Landscape of Religion, New York: Oxford University Press.

Barrett, J.L. (2004), 'Counterfactuality in counterintuitive religious concepts', Behavioral \& Brain Sciences, 27, pp. 731-32.

Barrett, J.L. and Nyhof, M.A. (2001), 'Spreading non-natural concepts: The role of intuitive conceptual structures in memory and transmission of cultural materials', Journal of Cognition \& Culture, 1, pp. 69-100.

Bloom, P. (2004), Descartes' Baby: How the Science of Child Development Explains What Makes us Human, New York: Basic Books.

Boyer, P. (2001), Religion Explained: The Evolutionary Origins of Religious Thought, New York: Basic Books.

Boyer, P. and Ramble, C. (2001), 'Cognitive templates for religious concepts: Crosscultural evidence for recall of counter-intuitive representations', Cognitive Science, 25, pp. 536-564. (2003), 'Religious Thought and Behavior as By-products of Brain Function', Trends in Cognitive Sciences, 7, pp. 119-24.

Buss, D.M. (1989), 'Sex differences in human mate preferences: evolutionary hypotheses tested in 37 cultures', Behavioral and Brain Sciences, 12, pp. 1-49.

Cohen, E. (2007), The Mind Possessed: The Cognition of Spirit Possession in an AfroBrazilian Religious Tradition, Oxford: Oxford University Press. 
Cooper, A. and Sportolari, L. (1997), 'Romance in cyberspace: Understanding online attraction', Journal of Sex Education and Therapy, 22: 1, pp. 7-14.

Dunbar, R.I.M. (2004), The Human Story: A New History of Mankind's Evolution, London: Faber and Faber.

Ellis, Bruce J. (1992), 'The evolution of sexual attraction: Evaluative mechanisms in women', in J.H. Barkow, L. Cosmides and J. Tooby (eds), The Adapted Mind: Evolutionary Psychology and the Generation of Culture, New York: Oxford University Press, pp. 267-88.

Fiske, A.P. (2002), 'Socio-moral emotions motivate action to sustain relationships', Self and Identity, 1, pp. 169-75.

Fessler, D.M.T. and Haley, K.J. (2003), 'The strategy of affect: Emotions in human cooperation', in P. Hammerstein (ed.), The Genetic and Cultural Evolution of Cooperation, Cambridge, MA: MIT Press, pp. 7-36.

Gambetta, D. (1994), 'Godfather's gossip', Archives Europeennes de Sociologie, 35, pp. 199-223.

Garau, M., Slater, M., Pertaub, D.P. and Razzaque, S. (2005), 'The responses of people to virtual humans in an immersive virtual environment', Presence-Teleoperators and Virtual Environments, 14: 1, pp. 104-16.

Gazzaniga, M.S., Velletri, A.S. and Premack, D. (1971), 'Language training in braindamaged humans', Federation Proceedings, 30, p. 265.

Gibson, W. (1984), Neuromancer, New York: Ace. 
Grammer, K. and Thornhill, R. (1994), 'Human (Homo sapiens) facial attractiveness and sexual selection: The role of symmetry and averageness', Journal of Comparative Psychology, 108, pp. 233-42.

Grossman, C.L. (2007), 'Faithful Build a Second Life for Religion Online', http://www.usatoday.com/tech/gaming/2007-04-01-second-life-religion_N.htm. Accessed 27 April 2008.

Harris, R.J., Schoen, L.M. and Hensley, D.L. (1992), 'A cross-cultural study of story memory', Journal of Cross-Cultural Psychology, 23, pp. 133-47.

Haviland, J.B. (1977), Gossip, Reputation, and Knowledge in Zincantan, Chicago: University of Chicago Press.

Hertenstein, M.J., Holmes, R.M., Kerestes, A.M. and Verkamp, J.M. (2006), 'The communicative functions of touch in humans, nonhuman primates, and rats: A review and synthesis of the empirical research', Genetic, Social, and General Psychology Monographs, 132: 1, pp. 5-94.

Hornbeck, R.G. (2007), 'Virtual touch and affection in SL', unpublished M.Sc. thesis, Oxford University, Oxford.

Horton, C. (2007), 'Cyberspankings: The appeal of virtual S \& M', Pixel Pulse Magazine, http://www.slpixelpulse.com/2007/02/09/cyberspankings-the-appeal-ofvirtual-sm/. Accessed 8 August 2007.

Imai, S. and Richman, C.L. (1991), 'Is the bizarreness effect a special case of sentence reorganization?', Bulletin of the Psychonomic Society, 29, pp. 429-32.

Johnson, L. (2007), 'Down the Rabbit Hole ... or how the NMC took the red pill, got a 
SL, and found love on the 3D Web', http://immersiveeducation.org/library/Down the Rabbit Hole-Larry Johnson 2007.pdf. Accessed 8 August 2007.

Keil, F.C. (1989), Concepts, Kinds, and Cognitive Development, Cambridge, MA: MIT Press.

Kelly, M. and Keil, F.C. (1985), 'The more things change...: Metamorphoses and conceptual structure', Cognitive Science, 9, pp. 403-16.

Lattin, D. (moderator) (2007), 'This revolution will be televised from SL', News 21: Faces of Faith in America, 2 August, http://newsinitiative.org/story/2007/07/31/this revolution will be televised. Accessed 20 September 2007.

Lea, M., Spears, R. and DeGroot, D. (1995), 'Love at first byte? Building personal relationships over computer networks', in J.T. Wood and S.W. Duck (eds), Understudied Relationships: Off The Beaten Track, Newbury Park: Sage, pp. 197-233.

Lewis, C.S. (1952), The Voyage of the Dawn Treader, England: Geoffrey Bles.

Machalinski, A., McConnon, A. and Nicholson, C. (2006), 'Dating and mating in Second Life', http://www.scienceandsex.com/images/TheDigitalLover.pdf. Accessed 8 August 2007.

McCauley, R.N. (forthcoming), The Naturalness of Religion and the Unnaturalness of Science.

Pesce, M. (1997), 'Ritual and the virtual', in R. Ascott (eds.), Consciousness Reframed: Abstracts, Newport: CAiiA, University of Wales College.

Pinker, S. (1997), How The Mind Works, London: Penguin. 
Povinelli, D. J. and Vonk, J. (2003), 'Chimpanzee minds: suspiciously human?’, Trends in Cognitive Sciences, 7(4), pp. 157-160.

Rogers, K. (1997), 'Viperscience', in R. Ascott (ed.), Consciousness Reframed: Abstracts, Newport: CAiiA, University of Wales College.

Rubin, D.C. (1995), Memory in Oral Traditions: The Cognitive Psychology of Epics, Ballads, and Counting-Out Rhymes, New York: Oxford University Press.

Rumelhart, D.E. (1997), 'Understanding and summarizing brief stories', in D. LaBerge and S.J. Samuels (eds), Basic Processes in Reading: Perception and Comprehension, Hillsdale, NJ: Lawrence Erlbaum Associates.

Schmidt, S.R. (1991), 'Can we have a distinctive theory of memory?', Memory and Cognition, 19, pp. 523-42.

Spelke, E.S. and Kinzler, K.D. (2007), 'Core knowledge', Developmental Science, 11, pp. 89-96.

Sperber, D. (1996), Explaining Culture: A Naturalistic Approach, Oxford: Blackwell.

Sperber, D. and Hirschfeld, L.A. (2003), 'The cognitive foundations of cultural stability and diversity', Trends in Cognitive Sciences, 8, pp. 40-46.

Sperber, D., Premack, D. and Premack, A. J. (eds) (1995), Causal Cognition: A Multidisciplinary Debate, New York: Oxford University Press, pp. 44-78.

Sperber, D. and Wilson, D. (1995), 'Relevance: Communication and Cognition, Oxford: Blackwell. 
Springer, K. and Keil, F.C. (1989), 'On the development of biologically specific beliefs: The case of inheritance', Child Development, 60, pp. 637-84.

Stiller, J., Nettle, D. and Dunbar, R. (2004), 'The small world of Shakespeare's plays', Human Nature, 14, pp. 397-408.

Thorndyke, P.W. (1977), 'Cognitive structures in comprehension and memory of narrative discourse', Cognitive Psychology, 9, pp. 77-110.

Tomasello, M., Call, J., and Hare, B. (2003), 'Chimpanzees understand psychological states - the question is which ones and to what extent', Trends in Cognitive Sciences, 7(4), pp. 153-156.

Uvnas-Moburg, K. (1998), 'Oxytocin may mediate the benefits of positive social interaction and emotions', Psychoneuroendocrinology, 8, pp. 819-35. (2003), The Oxytocin Factor, Cambridge, MA: Da Capo Press.

Viklund, A. (2007), 'Hello hello!', http://virtualanalise.wordpress.com/2007/05/. Accessed 8 August 2007.

Waddill, P.J. and McDaniel, M.A. (1998), 'Distinctiveness effects in recall: Differential processing or privileged retrieval?', Memory and Cognition, 26, pp. 108-20.

Ward, T.B. (1994), 'Structured imagination: The role of category structure in exemplar generation', Cognitive Psychology, 27, pp. 1-40. (1995), 'What's old about new ideas?', in S.M. Smith, T.B. Ward and R.A. Finke (eds), The Creative Cognitive Approach, Cambridge, MA: MIT Press, pp. 157-78. 
Wertheim, M. (1999), 'Is Cyberspace a Spiritual Place?' Cybersociology Magazine, 7 (1 September), http://www.cybersociology.com/files/7 wertheim.html. Accessed 10 October 2007.

Yee, N. (2005), 'In their own words: The immersion component', http://www.nickyee.com/daedalus/archives/001302.php. Accessed 8 August 2007.

\begin{abstract}
Virtual reality (VR) is often described as a gateway to a religious or spiritual experience - but why? In this article, using theories and evidence taken from the cognitive science of religion (CSOR), we hypothesize that human minds may interact with $V R$-hosted phenomena in a manner highly similar to that in which they interact with supernatural concepts. Specifically, we note that both VR inputs and supernatural concepts contain information that (1) contradicts the intuitive set of expectations we bring to an ontological category of phenomena (for example, natural objects, animals) and (2) allows us to draw a superabundance of inferences from our social cognitive mechanisms with minimal effort. We then summarize these points by illustrating a common VR phenomenon - 'virtual touch' - wherein counterintuitive representations and strategic information coalesce to create an emotionally salient experience that is itself counterintuitive and by some accounts spiritual-like.
\end{abstract}

